\title{
Effect of pregabalin on nociceptive thresholds and immune responses in a mouse model of incisional pain
}

\author{
Jung Hyun Park ${ }^{1}$, Seung Hee $\mathrm{Cho}^{2}$, Rip Kim², Sang Hoon $\mathrm{Na}^{2}$, Eun-sun Kang ${ }^{3}$, Mi-young Yeom ${ }^{3}$, and Yeon Jang ${ }^{1}$ \\ 'Department of Anesthesiology and Pain Medicine, Incheon St. Mary's Hospital, College of Medicine, The Catholic University of Korea, Incheon, Korea \\ ${ }^{2}$ Department of Anesthesiology and Pain Medicine, Catholic Medical Center, College of Medicine, The Catholic University of Korea, Seoul, Korea \\ ${ }^{3}$ Institute for Bio-Medical Convergence, The Catholic University of Korea, Incheon St. Mary's Hospital, Incheon, Korea
}

Received October 14, 2020

Revised December 26, 2020

Accepted December 28, 2020

Handling Editor: Jong Yeon Park

\section{Correspondence}

Yeon Jang

Department of Anesthesiology and Pain Medicine, Incheon St. Mary's Hospital, College of Medicine, The Catholic University of Korea, 56 Dongsu-ro, Bupyeong-gu, Incheon 21431, Korea Tel: +82-32-280-5992

Fax: +82-32-280-5416

E-mail: anesjang@catholic.ac.kr

Seung Hee Cho's current affiliation: Department of Anesthesiology and Pain Medicine, Incheon St. Mary's Hospital, College of Medicine, The Catholic University of Korea, Incheon, Korea
Background: It is known that some analgesics as well as pain can affect the immune system. The aim of this study was to investigate the analgesic effect and immunomodulation of pregabalin (PGB) in a mouse incisional pain model.

Methods: A postoperative pain model was induced by hind paw plantar incision in male BALB/c mice. Mice were randomly divided into four groups ( $n=8)$ : a salinetreated incision (incision), PGB-treated incision (PGB-incision), sham controls without incision or drug treatment (control), and a PGB-treated control (PGB-control). In the PGB treated groups, PGB was administered intraperitoneally (IP) 30 minutes before and 1 hour after the plantar incision. Changes of the mechanical nociceptive thresholds following incision were investigated. Mice were euthanized for spleen harvesting 12 hours after the plantar incision, and natural killer (NK) cytotoxicity to YAC 1 cells and lymphocyte proliferation responses to phytohemagglutinin were compared among these four groups.

Results: Mechanical nociceptive thresholds were decreased after plantar incision and IP PGB administration recovered these decreased mechanical nociceptive thresholds $(P<0.001)$. NK activity was increased by foot incision, but NK activity in the PGB-incision group was significantly lower than that in the Incision group $(P<$ 0.001). Incisional pain increased splenic lymphocyte proliferation, but PGB did not alter this response.

Conclusions: Incisional pain alters cell immunity of the spleen in BALB/c mice. PGB showed antinocieptive effect on mouse incisional pain and attenuates the activation of NK cells in this painful condition. These results suggest that PGB treatment prevents increases in pain induced NK cell activity.

Key Words: Analgesics; Immunity, Cellular; Immunomodulation; Killer Cells, Natural; Mice; Pain, Postoperative; Pregabalin; Spleen.

\section{INTRODUCTION}

Surgical trauma and the accompanying pain are associated with postoperative alterations of the neuroendocrine, metabolic, and immune systems $[1,2]$. Proper postopera- tive pain management can prevent these alterations and reduce the cardiopulmonary and metabolic complications. Therefore, the use of appropriate analgesics helps improve the outcome of surgery [3]. On the other hand, it is known that some anesthetics and analgesics, apart from (c) This is an open-access article distributed under the terms of the Creative Commons Attribution Non-Commercial License (http://creativecommons.org/licenses/by-nc/4.0/), which permits unrestricted non-commercial use, distribution, and reproduction in any medium, provided the original work is properly cited.

(C) The Korean Pain Society, 2021
Author contributions: Jung Hyun Park: Writing/manuscript preparation; Seung Hee Cho: Computation; Rip Kim: Computation; Sang Hoon Na: Formal analysis; Eun-sun Kang: Investigation; Mi-young Yeom: Investigation; Yeon Jang: Study conception. 
their analgesic effects, themselves have immunosuppressive effects. This means that the choice of analgesic drug is important in immunocompromised patients [4-7].

The antiepileptic drug pregabalin (PGB) has been used extensively to treat various types of neuropathic pain, including diabetic polyneuropathy and post-herpetic neuralgia [8]; it also reduces acute and chronic postoperative pain and postoperative opioid consumption $[9,10]$. We previously examined the effects of PGB treatment on immune reactions in chronic neuropathic mice. In that study, mice with chronic neuropathy, due to constriction of the sciatic nerve, exhibited immune upregulation and PGB treatment reversed this [7]. However, little further is known about the immunological effects of PGB administration in acute pain or painful postoperative conditions and the advantages of perioperative PGB treatment remain controversial [10].

The aim of the present study was to assess whether PGB treatment affects the immune reactions associated with postoperative pain. To investigate cell-mediated immune responses, we employed an incisional pain model in mice and evaluated the effects of PGB on natural killer (NK) cell activity and proliferation of splenic lymphocytes. At the same time, we assessed the antinociceptive effect of PGB using the paw withdrawal threshold (PWT) test. The mouse pain model devised by Pogatzki and Raja [11] is a modified mouse murine version of a well-established rat model of incisional pain. Pogatzki et al. [12] suggested that ongoing afferent input from the plantar incision wound in this model maintains sensitization of the dorsal horn neurons. As a result, the mechanical and heat hyperalgesia continue for several days which is similar with variable surgical pain in humans.

\section{MATERIALS AND METHODS}

\section{Animal use}

All experiments were approved by the Institutional Animal Care and Use Committee (Incheon St. Mary's Hospital, College of Medicine, The Catholic University of Korea, Permit Number was CIMH 2012-001). All procedures were conducted in accordance with the guidelines specified in the National Institutes of Health (NIH) Guide for the Care and Use of Laboratory Animals (NIH publication no. 86-23, revised 1985) as well as the Ethical Guidelines for Investigations of Experimental Pain in Conscious Animals. The experiments were performed on 50 male BALB/ c mice (Orient Bio, Seoul, Korea) weighing 20 to $25 \mathrm{~g}$ and about 6 weeks of age. Mice were housed in groups of five per cage under standard conditions (i.e., a room tempera- ture of $20^{\circ} \mathrm{C}$ to $25^{\circ} \mathrm{C}$, relative humidity of $50 \%$ to $60 \%$, and a 12/12 hr light/dark cycle, with the lights on at 08:00) and all experiments were performed at the animal laboratory between $7 \mathrm{am}$ and $11 \mathrm{am}$. The mice were kept specific pathogen-free and allowed access to food and water ad libitum throughout the study. The mice were acclimatized for at least 1 week before the study.

\section{Hind paw plantar incision}

As an acute pain model, we used the mouse model of plantar incisional pain [11]. Mice were anesthetized with isoflurane $2 \%-3 \%$ that was delivered through a nose cone. After antiseptic preparation of the left hind paw with alcohol, a No. 11 blade was used to make a $5 \mathrm{~mm}$ longitudinal incision on the surface of the plantar foot that traversed the skin and fascia. The underlying muscle was elevated with curved forceps. The muscle origin and insertion were left intact. The skin was closed with a single mattress suture by using a TG175-8 needle with 6-0 nylon (Ophthalmic, 1716G: Ethicon, Somerville, NJ). Mice of the control group underwent anesthesia without plantar incision. All surgeries were performed by the same person to minimize experimental variability.

\section{Experimental setting and drug treatment}

Mice were randomized using block randomization into one of four groups (each $n=8$ ), as follows: saline-treated hind paw plantar incision (the incision group); PGBtreated incision (the PGB-incision group); sham controls undergoing an identical anesthesia procedure but without incision or administration of drugs (the control group); and PGB-treated controls (the PGB-control group). In the PGB-incision group, PGB in saline was administered intraperitoneally at a dose of $30 \mathrm{mg} / \mathrm{kg}$ in a volume of 0.1 $\mathrm{mL} / 10 \mathrm{~g}$. In the incision group, the same volume of saline was injected intraperitoneally. For the extension of analgesic effect of PGB without deep sedation, the drugs were administered divided into two doses. The first intraperitoneal injection of PGB or saline was performed 30 minutes before the plantar incision. After the surgery, mice were allowed to recover for 30 minutes before testing for postoperative mechanical thresholds. One hour after the hind paw incision, a second intraperitoneal PGB injection was performed with the same dosage. Mice were euthanized by cervical dislocation under isoflurane anesthesia 12 hours after the plantar incision, and NK cytotoxicity and lymphocyte proliferation were measured by a person who was blinded to the procedures in these animals (Fig. 1). 


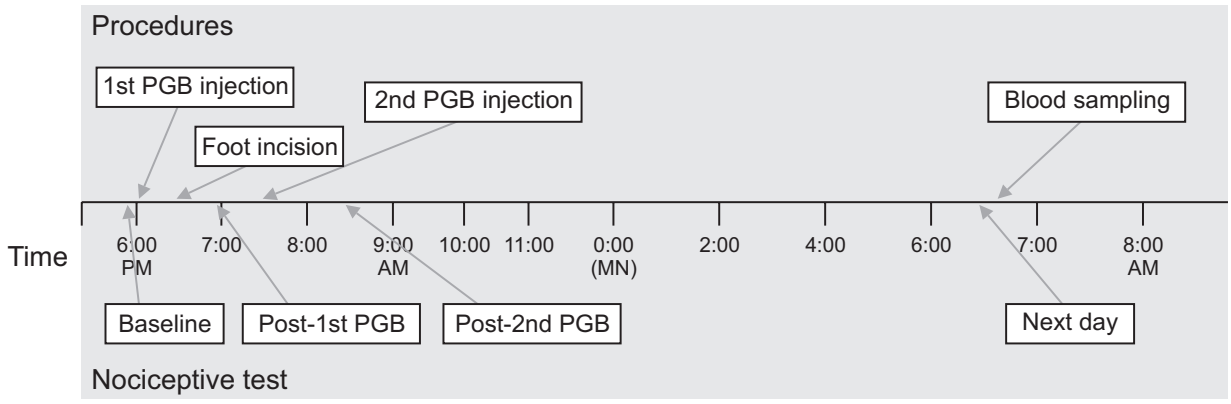

Fig. 1. Experimental procedure. At the beginning of the experiment, initial paw withdrawal threshold were assessed for mechanical nociceptive test (baseline). In the pregabalin (PGB)-incision and PGB-control groups, PGB in saline was administered intraperitoneally, at $30 \mathrm{mg} / \mathrm{kg}$, in a volume of 0.1 $\mathrm{mL} / 10 \mathrm{~g}$. In the incision group, an identical volume of saline was injected intraperitoneally. Thirty minutes subsequent to the initial drug injection, plantar incision or anesthesia was applied in accordance with group. One hour following hind paw incision, a second intraperitoneal injection was performed at the same dosage. Mice were sacrificed by cervical dislocation 12 hours following plantar incision, and natural killer cytotoxicity and lymphocyte proliferation were measured. The figure shows the whole experimental process using group 3 as an example.

\section{Nociceptive testing}

To determine the mechanical allodynia caused by the incision and the analgesic effect of preabalin on the pain, the mechanical nociceptive thresholds of the incision group and the PGB-incision group were compared with the control group. Mechanical nociceptive thresholds were assessed by PWT 30 minutes before plantar incision, 1 hour after the first and second PGB administrations, and 12 hours after surgery (the second day). The tests were performed by an investigator who was blinded to the drug or procedure that the mice had received. A dynamic plantar aesthesiometer (Ugo Basile, Gemonio, Italy) was used to measure the mechanical thresholds. Animals were placed individually in a test cage with a wire-mesh floor. A rigidtipped filament was applied to the skin of the mid-plantar area of the hind paw. The filament was exerted with an increasing force, starting below the threshold of detection and increasing to $5 \mathrm{~g}$ in 20 seconds, until the animal removed its paw. The PWT was expressed in grams (g). All measurements were performed in triplicate on the same hind paw at 30 second intervals. The mechanical threshold was defined as the mean value.

\section{Cell preparation}

Mice were euthanized by cervical dislocation under deep isoflurane anesthesia (3\%-4\%) and the spleen was aseptically excised and stored in tissue culture medium (RPMI 1640; Gibco BRL, Grand Island, NY). A single-cell suspension was prepared by using a $5 \mathrm{~mL}$ syringe plunger to pass spleen tissue in fresh wash medium through a $70 \mu \mathrm{m}$ mesh strainer (BD Falcon, San Diego, CA). The splenocytes were suspended in complete RPMI medium (RPMI 1640 supplemented with $10 \%$ fetal bovine serum, $100 \mathrm{U} / \mathrm{mL}$ penicillin, $100 \mu \mathrm{g} / \mathrm{mL}$ streptomycin, and $2 \mathrm{mM}$ L-glutamine). Cell density and viability were estimated with a hemocytometer. Trypan blue exclusion was performed to identify the dead cells.

\section{NK cell cytotoxicity assay}

A lactate dehydrogenase (LDH) assay with YAC-1 (mouse lymphoma cells) targets was conducted to determine the effects of incisional pain and of PGB treatment on the tumoricidal activity of NK cells from the spleen of each mouse. Target YAC-1 lymphoma cells were grown in RPMI 1640 medium supplemented with antibiotics and fetal bovine serum. Effector splenocyte suspensions were prepared from each mouse, and various numbers of effector cells were added to the wells of a microtiter plate containing $1 \times 10^{4}$ target YAC-1 cells in $100 \mu \mathrm{L}$ to achieve final effector to target cell ratios of 80:1, 40:1, and 20:1. The plates were incubated for 4 hours at $37^{\circ} \mathrm{C}$ in $5 \% \mathrm{CO}_{2}$ at $90 \%$ humidity. The cells were then removed by centrifugation, and the supernatants were collected and transferred to a flat-bottom plate. After $100 \mu \mathrm{L}$ of freshly prepared LDH detection mixture was added to each well, the plate was incubated at room temperature for 30 minutes to permit color development. The absorbance in each well was measured at $490 \mathrm{~nm}$ with a plate reader, and the percentage of specific LDH release was determined according to the following equation:

$$
\% \text { Specific lysis }=\frac{\mathrm{LDH}_{\text {experimental }}-\mathrm{LDH}_{\text {effector cells }}-\mathrm{LDH}_{\text {spontaneous }}}{\mathrm{LDH}_{\text {maximal }}-\mathrm{LDH}_{\text {spontaneous }}} \times 100
$$

where $\mathrm{LDH}_{\text {experimental }}=\mathrm{LDH}$ release from coculture of effector cells and target cells; $\mathrm{LDH}_{\text {effector cells }}=\mathrm{LDH}$ release from effector cells cultured on their own; $\mathrm{LDH}_{\text {spontaneous }}=$ LDH release from YAC-1 cells cultured on their own (low control); and $\mathrm{LDH}_{\text {maximal }}=\mathrm{LDH}$ release when YAC-1 cells 
were lysed in Triton X-100 (high control).

\section{Splenic lymphocyte proliferation activity assay}

The proliferative response of isolated splenocytes to phytohemagglutinin (PHA) was measured using a bromodeoxyuridine (BrdU) detection ELISA kit (Roche Molecular Biochemicals, Mannheim, Germany). The splenocytes from each mouse were aliquoted into 96-well micro plates at $2 \times 10^{5}$ cells/well $(100 \mu \mathrm{L} /$ well $)$ and cultured at $37^{\circ} \mathrm{C}$ in $5 \%$ $\mathrm{CO}_{2}$ at $90 \%$ humidity in the presence of PHA for 2 days; $5 \mu \mathrm{g}$ PHA was added at the start of incubation and then again 24 hours later. For pulse labeling, $10 \mu \mathrm{L}$ BrdU was added to each well (10 $\mu \mathrm{M}$ final concentration) and the cells were incubated for another 18 hours. The labeling medium was removed using a needle, the cells were air-dried, and 200 $\mu \mathrm{L}$ FixDenant (Roche Diagnostics, Indianapolis, IN) agent was added to each well to fix the cells and denature the genomic DNA, thereby exposing the incorporated BrdU to immunodetection. After removing the fixative, an antiBrdU antibody (clone BMG 6H8, Fab fragment) was added to each well and the plate was incubated at room temperature for 90 minutes. After washing with phosphatebuffered saline and adding substrate (tetramethylbenzidine), the plate was incubated at $25^{\circ} \mathrm{C}$ for 20 minutes. The absorbance at $370 \mathrm{~nm}$ was then measured by using an automated plate reader. Proliferation was determined as the stimulation index (SI), calculated as follows:

$$
\mathrm{SI}=\mathrm{OD}_{\text {PHA-stimulated wells }} / \mathrm{OD}_{\text {unstimulated wells }}
$$

where $\mathrm{OD}=$ optical density.

\section{Measurement of the plasma concentration of PGB}

To determine whether the remaining plasma PGB that was not metabolized affected immune response (i.e., NK cytotoxicity and lymphocyte proliferation), plasma concentrations of PGB were measured in 3 separate groups of mice $(\mathrm{n}=6)$. Mice were subjected to intraperitoneal injection of $30 \mathrm{mg} / \mathrm{kg}$ PGB, anesthesia, plantar incision, and a second injection of PGB 90 minutes after the first, as described above (Fig. 1). Plasma samples were obtained from the orbital sinus of each mouse 2, 6, or 12 hours (depending on the assigned group) after the first PGB injection and placed in a tube with sodium heparin. Each sample was centrifuged and the plasma was harvested and stored at $-80^{\circ} \mathrm{C}$ until the analysis was performed. Plasma PGB concentrations were measured by validated high-performance liquid chromatography/mass spectrometry (HPLC; 1260 solvent delivery system/6460 tandem mass spectrometer, Agilent, Santa Clara, CA).

\section{Statistical analysis}

Data are reported as means \pm standard deviation. All results were analyzed using Sigma-Stat version 3.1 (SPSS Inc., Chicago, IL). Two-way repeated-measures analysis of variance (ANOVA; two-factor repletion) with pairwise multiple-comparisons (Holm-Sidak method) was used to analyze the results of the behavioral experiment. Two-way ANOVA and Bonferroni $t$-tests were performed to evaluate differences in NK cytotoxicity. One-way ANOVA and multiple comparison tests (Bonferroni $t$-tests) were used to evaluate differences in the splenocyte proliferative response of the different groups. The Kruskal-Wallis oneway ANOVA by ranks test was applied to compare the serum PGB concentrations at different time points. A value of $P<0.05$ was taken to indicate statistical significance.

\section{RESULTS}

\section{Effects of PGB treatment on PWT}

The PWT was decreased following plantar incision; reduced mechanical thresholds were maintained until the following morning in the incision group, compared with the pre-surgery baseline mechanical threshold $(P<0.001)$. Double intraperitoneal injection of PGB (30 min before and $1 \mathrm{hr}$ following incision, each at $30 \mathrm{mg} / \mathrm{kg}$ ) produced significant antinociceptive effects $(P<0.001)$, and elevation of the PWT, until the second day, compared with the incision group. There were no differences in the serial PWTs of the control and PGB-incision groups, following the first and second dose of PGB $(P=0.867$; Fig. 2$)$. During the experimental periods, except anesthesia and recovery periods, the mice maintained normal activity, posture, and appetite.

\section{Effects of PGB on NK cell cytotoxic activity}

On the second day, the tumoricidal activity of NK cells against NK-sensitive YAC-1 lymphoma cells from each mouse spleen was assessed. Results are displayed as plots of LDH release (Fig. 3). NK cell activity was significantly higher in the incision group than in the control group $(P$ $<0.001$ ). Perioperative intraperitoneal PGB treatment, in the PGB-incision group, significantly reduced NK activity compared with saline injection in the incision group. However, NK activity in the PGB treatment group (PGBincision) remained higher vs. the control group $(P<0.001)$. However, there was no significant difference in the NK cell activity of PGB-control group mice and the control group $(P$ $=0.925$; Fig. 3). 


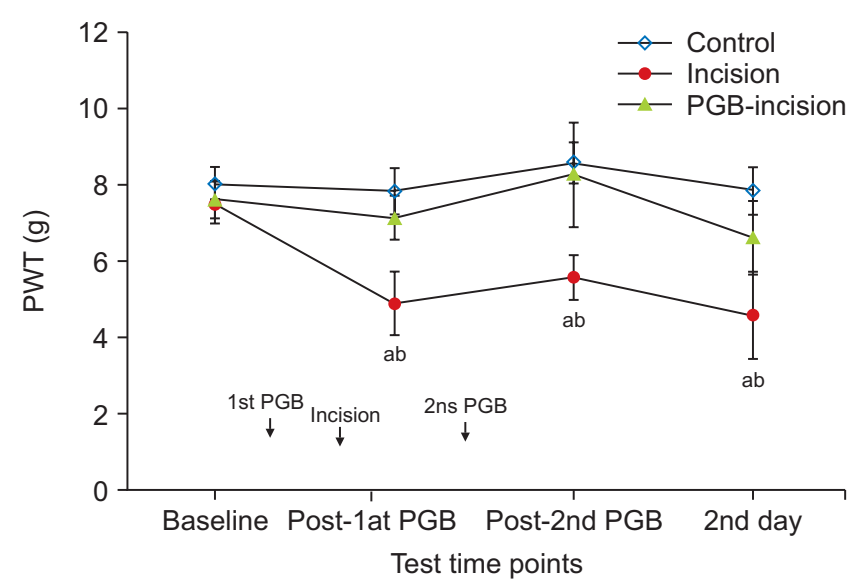

Fig. 2. Time course of paw withdrawal threshold (PWT) after plantar incision in mice ( $n=8$ per group). Foot incision resulted in the development of marked mechanical allodynia until the day after the incision in the incision group (saline + incision). Intraperitoneal injection of pregabalin (PGB) at $30 \mathrm{mg} / \mathrm{kg}$ significantly reduced mechanical allodynia in the PGB-incision group (PGB + incision). There were no significant differences in the PWT between the control and PGB-incision groups throughout the experiment. In the control and PGB-incision groups, there were no significant changes in the PWT compared with the baseline PWT. Data represent means \pm standard deviation. ${ }^{a} P<0.001$ vs. the control and PGB-incision groups. ${ }^{\mathrm{b}} P<0.001$ vs. baseline.

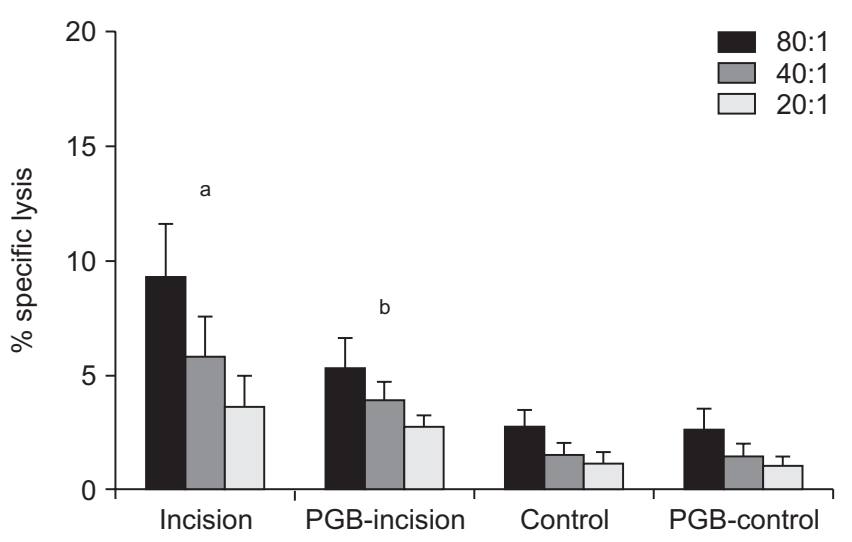

Fig. 3. Effects of perioperative pregabalin (PGB) treatment on natural killer (NK) cell activity (\% specific lysis) on the day after hind paw incision in mice ( $n=8$ per group). At various E:T ratios (80:1, 40:1, 20:1), the tumoricidal activities of NK cells (effector cells, E) against NK sensitive YAC-1 lymphoma cells (target cell, T) were calculated from lactate dehydrogenase release. NK cell activity was significantly increased in the incision group (saline + incision). In the PGB-incision group (PGB + incision), perioperative intraperitoneal $\mathrm{PGB}$ treatment significantly reduced NK activity compared with the incision group, but NK activity in the PGBincision group remained higher than in the control group $(P<0.001)$. Data represent means \pm standard deviation. ${ }^{\mathrm{a}} P<0.001$ vs. the PGBincision, control, and PGB-control groups. ${ }^{\mathrm{b}} P<0.001$ vs. the control and PGB-control groups.

\section{Effects of PGB on splenocyte proliferation}

The proliferative response of splenic lymphocytes to PHA

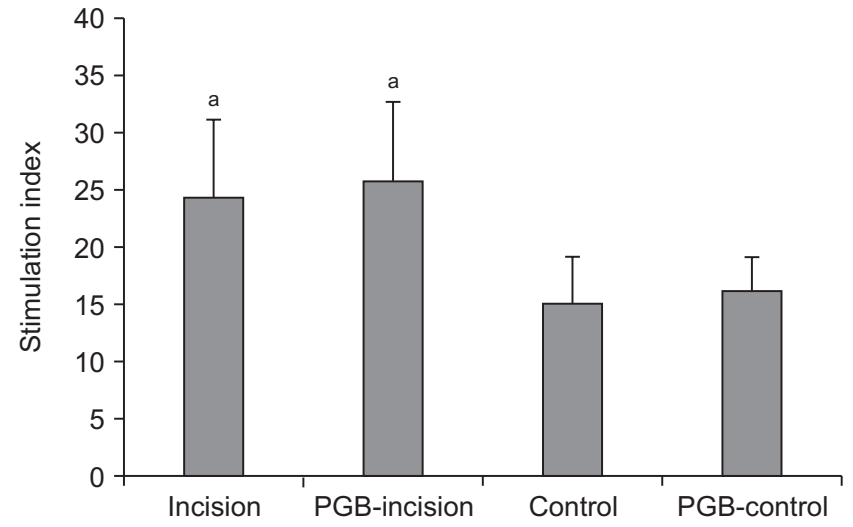

Fig. 4. Effects of perioperative pregabalin (PGB) treatment on splenocyte proliferation in mice the day following foot incision ( $n=8$ per group). Proliferation in response to $5 \mu \mathrm{g}$ of phytohemagglutinin mitogenic stimulation was determined as the percentage changes in the stimulation index. Incision-induced pain, in both the incision and PGB-incision groups, increased splenic lymphocyte proliferation to a significantly greater degree compared with the control group. However, PGB pretreatment in the PGB-control and PGB-incision groups did not induce greater changes in splenocyte proliferation compared with the control and incision groups, respectively. Data represent means \pm standard deviation. ${ }^{a} P<0.05$ vs. control.

is presented as a SI plot (Fig. 4). Incision-induced pain in both the incision and PGB-incision groups increased splenic lymphocyte proliferation to a greater degree compared with the control group $(P=0.012$ and $P=0.003$, respectively). However, there were no changes in the splenocyte proliferation responses of the incision group (24.38 $\pm 6.76 \%$ ) and PGB-incision group (25.77 $\pm 6.94 \%)$. Furthermore, PGB pretreatment in the PGB-control group did not induce significant changes in splenocyte proliferation compared with the control group $(P=0.786)$.

\section{PGB concentrations following intraperitoneal injection}

We measured the blood PGB concentrations in a separate groups of mice to rule out the possibility of direct effects of PGB on immune responses. Two hours subsequent to intraperitoneal PGB injection (two doses at $30 \mathrm{mg} / \mathrm{kg}$, with a 90-min interval), plasma concentrations were $7.704 \pm 0.963$ $\mu \mathrm{g} / \mathrm{mL}$, but decreased to $0.583 \pm 0.147 \mu \mathrm{g} / \mathrm{mL}$ (at $6 \mathrm{hr}$ following injection), and finally to $0.013 \pm 0.003 \mu \mathrm{g} / \mathrm{mL}$ (at 12 hr following injection; Fig. 5).

\section{DISCUSSION}

The present study showed that immediately after plantar incision, mechanical allodynia developed. This response was maintained until the termination of the experiment 12 


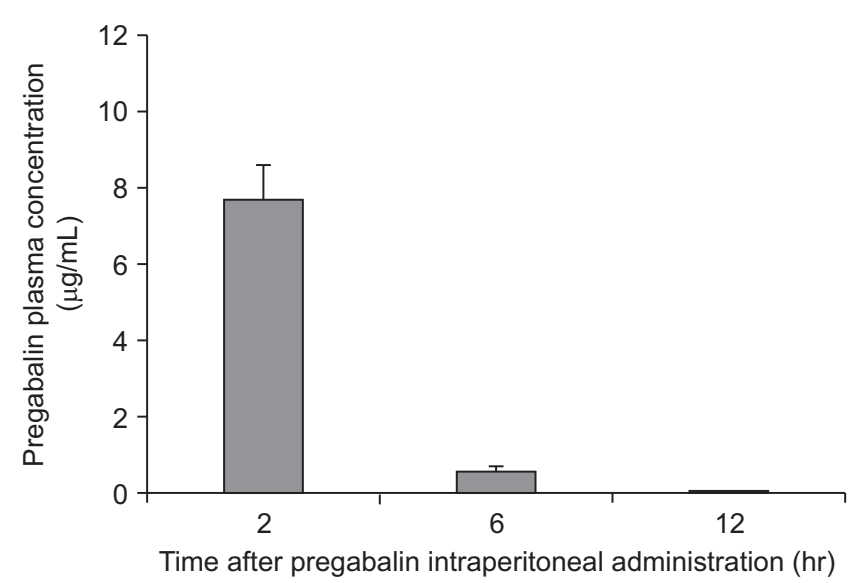

Fig. 5. Change in plasma pregabalin (PGB) concentration over time. In this auxiliary experiment, six mice underwent plantar incision and preoperative and postoperative PGB treatment, and blood was taken 2, 6, and 12 hours after the first PGB injection. Plasma PGB concentrations were measured by using high-performance liquid chromatography/mass spectrometry. Twelve hours after the first injection, which is when spleens were harvested for immune response analyses, the PGB concentration was close to zero $(0.013 \pm 0.003 \mu \mathrm{g} / \mathrm{mL})$. Data represent means \pm standard deviation.

hours after the incision was made. The incision increased NK cell activity and splenocyte proliferation. Perioperative PGB treatment attenuated this incision-induced NK cell activation to some degree. However, it did not influence the incision-induced splenocyte proliferation at all.

PGB is increasingly being seen as a useful adjunct in the multimodal management of postoperative pain. This is supported by the study of Field et al. [9], who showed, with a rat model of post-incisional pain, that preoperative administration of PGB may reduce the tissue damageinduced hyperexcitability of the dorsal horn neurons, thereby reducing both acute postoperative pain and the development of postoperative chronic pain. Moreover, a meta-analysis of clinical trials showed that perioperative PGB administration effectively reduces postoperative opioid consumption, especially after major surgery that induces severe pain $[10,13,14]$. Notably, it has been reported that depending on the type of pain, the analgesic dose of PGB varies from 10 to $>200 \mathrm{mg} / \mathrm{kg}$ [15]. Behavior observation studies in animals may be affected by sedation or decreased motor function caused by the administered drug, but previous studies have shown that PGB did not cause severe sedation or gait abnormalities at the analgesic dose [16].

In our study, $30 \mathrm{mg} / \mathrm{kg}$ PGB was administered twice (30 min before surgery and $1 \mathrm{hr}$ after surgery); we observed that the pain of the mice was effectively blocked despite the fact that the pain stimulus was ongoing. PGB-treated mice did not exhibit severe sedation. Moreover, during our preliminary experiment, we observed that the PWT of the PGB-control group was not different from the PWT of the control group. These results imply that the therapeutic dose of PGB in mice does not inhibit PWT in the painless state, which is consistent with a previous study by Takeuchi et al. [17].

Several studies have shown that severe or chronic pain in the postoperative period can have both immunostimulatory and immunosuppressive effects. Sacerdote et al. [18] showed that abdominal surgery for uterine cancer suppresses mitogen-induced T cell proliferation. Sharify et al. [19] found that mice given heat shocks exhibited upregulation of NK cell activity and splenocyte proliferation. Similarly, our previous study showed that when mice were subjected to chronic constriction injury of the sciatic nerve, their NK cell activity and splenocyte proliferation both rose [7]. Our present study observed similar effects from pain: we observed that plantar incision in mice significantly upregulated NK cell cytotoxic activity and mitogen-induced splenocyte proliferation. These findings probably reflect the fact that the immune and nervous systems are known to interact $[20,21]$. These immunomodulatory effects of pain can be harmful, especially if the pain is prolonged and severe, because it could limit the ability of the patient to resist infection [22]. Consequently, it is important to properly manage severe postoperative pain.

However, this approach also has its pitfalls because some analgesic drugs can themselves significantly alter immune responses: several studies in humans and animals show that opiates such as morphine suppress NK cell activity, T cell responses in delayed type hypersensitivity, cytotoxic $\mathrm{T}$ cell activity, $\mathrm{T}$ cell proliferation to mitogens, phagocytosis, and chemotaxis $[18,20,23]$. Moreover, in the absence of pain, mice treated with tramadol show upregulation of NK cell cytotoxicity and mitogen-induced splenocyte proliferation [6], while Lewis rats treated with morphine exhibit marked downregulation of these responses [24]. Notably, however, our present and previous [7] studies both showed that PGB treatment had no effect on the NK cell cytotoxicity or mitogen-induced splenocyte proliferation of control (non-incised) mice. These discrepancies may reflect the varying effects of different analgesics.

Several studies show that analgesics can also alter the immune changes induced by pain [4-6]. In particular, our previous study showed that when mice with chronic constriction of the sciatic nerve were given twice-daily postoperative $30 \mathrm{mg} / \mathrm{kg}$ PGB treatment starting 2 days after surgery, their pain-upregulated NK cell activity and splenocyte proliferation 7 days after surgery were both significantly depressed [7]. Our present observations in our incision model differ somewhat: while pain increased both of these immune responses and PGB largely (but not completely) reversed the effect of pain on NK cell activity, 
PGB treatment had no effect on mitogen-induced splenocyte proliferation 12 hours after surgery. These differences may reflect the fact that our present and previous [7] studies employed different pain models and that the immune responses were observed at different times $(12 \mathrm{hr}$ and 7 days after surgery, respectively).

These observations led us to hypothesize that PGB has a positive immunomodulatory effect, namely, it significantly suppresses the immunostimulatory effects of pain on NK cell activity and, depending on the type of pain, lymphocyte proliferation. This is important because patients with chronic neuropathic pain often require long-term use of PGB, and PGB is commonly prescribed as a supplement to opioids, which have immunosuppressive effects [10]: it is possible that PGB may ameliorate the pain-induced alterations in immune functions, although this requires further research. These findings together also suggest that the immunomodulatory effects of pain and analgesics depend on the nature and duration of the stressor that is applied, the immunological variables that are being investigated, and the analgesic that is used [19].

To determine whether the immune responses we observed 12 hours after PGB injection was directly affected by the PGB in the plasma, we examined the changes in PGB plasma concentrations 2, 6, and 12 hours after the intraperitoneal injection of this analgesic. The blood PGB concentration was close to zero at the 12 hours timepoint. Moreover, our previous in vitro study showed that $3 \mu \mathrm{g} / \mathrm{mL}$ PGB does not decrease NK cell cytotoxic activity [7]. Thus, it seems that the immunomodulatory effects of PGB on NK cell activity are not a direct consequence of the drug remaining in plasma; rather, it may be a pharmacodynamic consequence of the analgesic effect of the drug during the early postoperative period.

How pain activates NK cell functions is not clear. However, because lymphocytes express variable hormone receptors, it has been suggested that hormones mediate stress-induced changes in immune function. In particular, catecholamines seem to play an important role in the endocrine-neural-immune network $[25,26]$. Interestingly, a study by Takeuchi et al. [17] showed that intra-cisternal administration of catecholaminergic neurotoxin 6-hydroxydopamine hydrobromide to decrease the central noradrenalin attenuated the analgesic effect of PGB. How PGB reverses the upregulatory effect of pain on NK cell activity is unclear. However, it is thought that the analgesic effects of PGB involve a supraspinal mechanism that affects the locus coeruleus neurons that associate with the descending noradrenergic inhibitory system and spinal adrenergic receptors $[17,27]$.

Thus, this supraspinal mechanism may also mediate the immunomodulatory effect of PGB. Our study provides the framework for further investigations on the immunomodulatory effects of PGB. Future studies will aim to identify the multiple factors that affect immune responses to pain and PGB. These studies will include other types of pain, its effects on other immune responses, and other PGB treatment regimens. How PGB interacts with other analgesics in terms of its immunomodulatory effects should be assessed. In summary, foot incision in mice not only generated mechanical allodynia, it also increased NK cell cytotoxic activity and lymphocyte proliferation. Perioperative PGB treatment largely prevented the pain-induced increase in NK cell activity. However, these effects of PGB on NK cell activity were not evident in the absence of incisional pain.

In conclusion, this study suggests that PGB may be able to modulate the immune response that accompanies tissue injury and postoperative pain. As a drug used to treat chronic pain patients, these immunomodulatory effects are of great clinical importance and deserve further study.

\section{CONFLICT OF INTEREST}

No potential conflict of interest relevant to this article was reported.

\section{FUNDING}

No funding to declare.

\section{ORCID}

Jung Hyun Park, https://orcid.org/0000-0001-6376-3433

Seung Hee Cho, https://orcid.org/0000-0003-3336-7717

Rip Kim, https://orcid.org/0000-0002-3893-4947

Sang Hoon Na, https://orcid.org/0000-0002-8507-7466

Eun-sun Kang, https://orcid.org/0000-0003-4261-1320

Mi-young Yeom, https://orcid.org/0000-0002-9525-6441

Yeon Jang, https://orcid.org/0000-0002-2078-6149

\section{REFERENCES}

1. Kennedy BC, Hall GM. Neuroendocrine and inflammatory aspects of surgery: do they affect outcome? Acta Anaesthesiol Belg 1999; 50: 205-9.

2. Kiecolt-Glaser JK, Page GG, Marucha PT, MacCallum RC, Glaser R. Psychological influences on surgical recovery. Perspectives from psychoneuroimmunology. Am Psychol 1998; 53: 1209-18. 
3. Kehlet H, Holte K. Effect of postoperative analgesia on surgical outcome. Br J Anaesth 2001; 87: 62-72.

4. Tsai YC, Won SJ, Lin MT. Effects of morphine on immune response in rats with sciatic constriction injury. Pain 2000; 88: 155-60.

5. Beilin B, Shavit Y, Trabekin E, Mordashev B, Mayburd E, Zeidel A, et al. The effects of postoperative pain management on immune response to surgery. Anesth Analg 2003; 97: 8227.

6. Sacerdote P, Bianchi M, Manfredi B, Panerai AE. Effects of tramadol on immune responses and nociceptive thresholds in mice. Pain 1997; 72: 325-30.

7. Jang Y, Song HK, Yeom MY, Jeong DC. The immunomodulatory effect of pregabalin on spleen cells in neuropathic mice. Anesth Analg 2012; 115: 830-6.

8. van Seventer R, Bach FW, Toth CC, Serpell M, Temple J, Murphy TK, et al. Pregabalin in the treatment of post-traumatic peripheral neuropathic pain: a randomized double-blind trial. Eur J Neurol 2010; 17: 1082-9.

9. Field MJ, Holloman EF, McCleary S, Hughes J, Singh L. Evaluation of gabapentin and S-(+)-3-isobutylgaba in a rat model of postoperative pain. J Pharmacol Exp Ther 1997; 282: 12426.

10. Zhang J, Ho KY, Wang Y. Efficacy of pregabalin in acute postoperative pain: a meta-analysis. Br J Anaesth 2011; 106: 45462.

11. Pogatzki EM, Raja SN. A mouse model of incisional pain. Anesthesiology 2003; 99: 1023-7.

12. Pogatzki EM, Vandermeulen EP, Brennan TJ. Effect of plantar local anesthetic injection on dorsal horn neuron activity and pain behaviors caused by incision. Pain 2002; 97: 151-61.

13. Hegarty DA, Shorten GD. A randomised, placebo-controlled trial of the effects of preoperative pregabalin on pain intensity and opioid consumption following lumbar discectomy. Korean J Pain 2011; 24: 22-30.

14. Lam DMH, Choi SW, Wong SSC, Irwin MG, Cheung CW. Efficacy of pregabalin in acute postoperative pain under different surgical categories: a meta-analysis. Medicine (Baltimore) 2015; 94: e1944.

15. Chesler EJ, Ritchie J, Kokayeff A, Lariviere WR, Wilson SG, Mogil JS. Genotype-dependence of gabapentin and pregabalin sensitivity: the pharmacogenetic mediation of analgesia is specific to the type of pain being inhibited. Pain 2003; 106: 325-35.
16. Field MJ, Oles RJ, Lewis AS, McCleary S, Hughes J, Singh L. Gabapentin (neurontin) and S-(+)-3-isobutylgaba represent a novel class of selective antihyperalgesic agents. Br J Pharmacol 1997; 121: 1513-22.

17. Takeuchi Y, Takasu K, Ono H, Tanabe M. Pregabalin, S-(+)3 -isobutylgaba, activates the descending noradrenergic system to alleviate neuropathic pain in the mouse partial sciatic nerve ligation model. Neuropharmacology 2007; 53: 842-53.

18. Sacerdote P, Bianchi M, Gaspani L, Manfredi B, Maucione A, Terno G, et al. The effects of tramadol and morphine on immune responses and pain after surgery in cancer patients. Anesth Analg 2000; 90: 1411-4.

19. Sharify A, Mahmoudi M, Izad MH, Hosseini MJ, Sharify M. Effect of acute pain on splenic NK cell activity, lymphocyte proliferation and cytokine production activities. Immunopharmacol Immunotoxicol 2007; 29: 465-76.

20. Eisenstein TK, Hilburger ME. Opioid modulation of immune responses: effects on phagocyte and lymphoid cell populations. J Neuroimmunol 1998; 83: 36-44.

21. Ren K, Dubner R. Interactions between the immune and nervous systems in pain. Nat Med 2010; 16: 1267-76.

22. Zajączkowska R, Leppert W, Mika J, Kocot-Kępska M, Woroń J, Wrzosek A, et al. Perioperative immunosuppression and risk of cancer progression: the impact of opioids on pain management. Pain Res Manag 2018; 2018: 9293704.

23. Peterson PK, Molitor TW, Chao CC. Mechanisms of morphine-induced immunomodulation. Biochem Pharmacol 1993; 46: 343-8.

24. Lysle DT, Coussons ME, Watts VJ, Bennett EH, Dykstra LA. Morphine-induced alterations of immune status: dose dependency, compartment specificity and antagonism by naltrexone. J Pharmacol Exp Ther 1993; 265: 1071-8.

25. Mössner R, Lesch KP. Role of serotonin in the immune system and in neuroimmune interactions. Brain Behav Immun 1998; 12: 249-71.

26. Madden KS, Moynihan JA, Brenner GJ, Felten SY, Felten DL, Livnat S. Sympathetic nervous system modulation of the immune system. III. Alterations in T and B cell proliferation and differentiation in vitro following chemical sympathectomy. J Neuroimmunol 1994; 49: 77-87.

27. Tanabe M, Takasu K, Takeuchi Y, Ono H. Pain relief by gabapentin and pregabalin via supraspinal mechanisms after peripheral nerve injury. J Neurosci Res 2008; 86: 3258-64. 